\title{
Crossover from ionic hopping to nearly constant loss in the fast ionic conductor $\mathrm{Li}_{0.18} \mathrm{La}_{0.61} \mathrm{TiO}_{3}$
}

\author{
A. Rivera, ${ }^{1,2}$ C. León, ${ }^{1}$ J. Sanz, ${ }^{2}$ J. Santamaria, ${ }^{1}$ C. T. Moynihan, ${ }^{3}$ and K. L. Ngai ${ }^{4}$ \\ ${ }^{1}$ GFMC, Departamento de Fisica Aplicada III, Facultad de Fisica, Universidad Complutense Madrid, 28040 Madrid, Spain \\ ${ }^{2}$ Instituto de Ciencia de Materiales de Madrid (CSIC), Cantoblanco, 28049 Madrid, Spain \\ ${ }^{3}$ Materials Science and Engineering Department, Rensselaer Polytechnic Institute, Troy, New York 12180-3590 \\ ${ }^{4}$ Naval Research Laboratory, Washington, DC 20375-5320
}

(Received 17 August 2001; revised manuscript received 7 December 2001; published 10 June 2002)

\begin{abstract}
Electrical conductivity measurements of the fast ionic conductor $\mathrm{Li}_{0.18} \mathrm{La}_{0.61} \mathrm{TiO}_{3}$ have been conducted at temperatures ranging from 8 to $300 \mathrm{~K}$ and frequencies between $20 \mathrm{~Hz}$ and $5 \mathrm{MHz}$. A detailed analysis of the ac conductivity shows the existence of a crossover between two different regimes. At high temperatures and/or low frequencies correlated ion hopping is responsible for a power-law frequency dependent and thermally activated ac conductivity. On the other hand, at sufficiently low temperatures and/or high frequencies, the ions do not have enough thermal energy or time to hop between neighboring sites, and remain caged. The ac conductivity is then characterized by a linear frequency dependence (i.e., the equivalent of a nearly constant loss) and by a weak exponential temperature dependence of the form $\exp \left(T / T_{0}\right)$. A crossover between the two regimes is found, which is thermally activated with an activation energy $E=0.17 \mathrm{eV}$, significantly lower than that observed for the dc conductivity, $E_{\sigma}=0.4 \mathrm{eV}$. From this result, it is shown that the so-called "augmented Jonscher expression" fails to describe the ac conductivity in the whole frequency and temperature ranges. All these findings suggest that the nearly constant loss originates from electrical loss occurring during the time regime while the ion is still confined in the potential-energy minimum. Further, it is proposed that the loss mechanism involves some type of process where the potential-energy minimum relaxes in time on a time scale much shorter than the ionic hopping time scale. At longer times, as soon as the ion has significant probability of being thermally activated out of the potential well, the nearly constant loss terminates and correlated ion hopping becomes the only contribution to the ac conductivity.
\end{abstract}

DOI: 10.1103/PhysRevB.65.224302

PACS number(s): $66.30 . \mathrm{Hs}$

\section{INTRODUCTION}

More than two decades ago, Wong and Angell ${ }^{1}$ pointed out that the real part of the electrical ac conductivity of ionic conductors seemed to show a linear frequency-dependence in the limit of low temperatures and high frequencies. This linear frequency dependent term, of the form $\sigma^{\prime}(\omega) \approx A \omega$, results in a nearly constant loss (NCL) in the dielectric loss spectra, $\varepsilon^{\prime \prime}(\omega)=\sigma^{\prime}(\omega) / \omega \approx A$. Lee et $_{\text {al. }}{ }^{2}$ later observed this behavior in a variety of ionic conductors, and proposed it to be a universal feature of these materials. As a universal property, it might be of fundamental interest in order to understand ion dynamics in ionically conducting materials at short times. However, there have been only a few investigations of the nature of this nearly constant loss, and very few experimental data showing this behavior. ${ }^{3}$ Similarly, most research activity in the field of ionic conductors ${ }^{4-14}$ has focused on the long-range ion motion occurring at higher temperatures, where the frequency dependence of the ionic conductivity was usually described by the Jonscher expression ${ }^{15}$

$$
\sigma^{\prime}(\omega) \approx \sigma_{0}\left[1+\left(\omega / \omega_{o}\right)^{n}\right]
$$

In this temperature range, a dc conductivity $\sigma_{0}$ is found in the limit of low frequency, and a power-law behavior is observed with a fractional exponent $n$ above a characteristic frequency $\omega_{o}$. Both $\sigma_{0}$ and $\omega_{o}$ are found to be thermally activated with about the same activation energy $E_{\sigma}$. This has been interpreted in different theoretical models ${ }^{15-20}$ as indicating that the ac conductivity given by Eq. (1) originates from a migration of ions by hopping between neighboring potential wells, which eventually gives rise to a dc conductivity at the lowest frequencies. Interestingly, it is usually assumed in the literature ${ }^{2,21-26}$ that the Jonscher conductivity and the linear frequency term (or NCL) are additive contributions, and that the total ac conductivity can be described by the so-called augmented Jonscher form

$$
\sigma^{\prime}(\omega)=\sigma_{0}\left[1+\left(\omega / \omega_{o}\right)^{n}\right]+A \omega .
$$

We proposed in a recent letter ${ }^{27}$ that the loss mechanism of the ion, while it is still confined in its potential-energy site ("cage"), may be the origin of the NCL observed in ionic conductors. At short times ions are vibrating in their potential energy wells of height $E_{m}$, with a frequency $\nu_{0}$ usually termed the attempt frequency. A microscopic residence time is defined $\tau_{m}=\nu_{0}^{-1} \exp \left(E_{m} / k_{B} T\right)$, such that, statistically, for times shorter than $\tau_{m}$ ions vibrate within their sites and for times longer than $\tau_{m}$ abandon the site by thermally activated hopping over the energy barrier. We found that the NCL is the dominant contribution to the ac conductivity at times shorter than $\tau_{m}$, i.e., at frequencies $\omega>\tau_{m}^{-1}$; therefore it is only observed at sufficiently high frequencies or low temperatures.

In this work, we present a more detailed experimental investigation of the crossover between the NCL and the ac conductivity region due to ionic hopping, by adding experimental data on the fast ionic conductor $\mathrm{Li}_{0.18} \mathrm{La}_{0.61} \mathrm{TiO}_{3}$. The choice of this ionically conducting material is based on its high ionic conductivity, ${ }^{27-31}$ and because it shows the highest 
value ever determined of the nearly constant loss, ${ }^{3}$ to our knowledge. By analyzing isothermal ac conductivity data, we extend the temperature range of the crossover region found in Ref. 27, obtaining the crossover temperatures at frequencies in the range $100 \mathrm{kHz}-3 \mathrm{MHz}$. The present results are in complete agreement with those previously published in the frequency range $100 \mathrm{~Hz}-100 \mathrm{kHz}$ from isochronal conductivity measurements. ${ }^{27}$

The NCL coefficient $A$ is found to be temperature dependent. Although the temperature dependence of $A$ can be described by an Arrhenius equation with a very small activation energy, the best fits are obtained using a weak exponential temperature dependence of the form $A \propto \exp \left(T / T_{0}\right)$. Finally, we show that the validity of Eq. (2) is at odds with the properties and nature of the crossover between the NCL and the ac conductivity due to ionic hopping, and, therefore, the total ac conductivity in ionic conductors may not be expressed by a simple addition of both contributions in the frequency domain. This is an important result, since it has an impact on the typical current interpretation of ac conductivity in ionic conductors, and suggests a serial picture in which the NCL ceases to exist when ions leave their cages and start hopping. All these results give further support to our interpretation of the NCL as originating from a loss mechanism while the ions are still confined within a cage, i.e., the probability of ion hopping to a neighboring site is very small.

\section{EXPERIMENT}

Samples were prepared by heating a stochiometric mixture of high-purity $\mathrm{LiCO}_{3}, \mathrm{La}_{2} \mathrm{O}_{3}$, and $\mathrm{TiO}_{2}$ reagents at $1350^{\circ} \mathrm{C}$ in air for $5-11 \mathrm{~h}$, and then quenching to room temperature. Sintered cylindrical pellets $1 \mathrm{~mm}$ thick and $5 \mathrm{~mm}$ in diameter, with evaporated silver electrodes, were used for electrical measurements. The Li content was determined by inductive coupled plasma (ICP) analysis. Impedance spectroscopy measurements were conducted in the frequency range $20 \mathrm{~Hz}-5 \mathrm{MHz}$ and for temperatures between 125 and $300 \mathrm{~K}$, using precision LCR meters HP4284A and HP4285A. In this temperature range, measurements were carried out under a $N_{2}$ flow to ensure an inert atmosphere. The temperature range was extended down to $8 \mathrm{~K}$ using a closed cycle helium refrigerator, with sample in vacuum, for frequencies in the range $100 \mathrm{~Hz}-100 \mathrm{kHz}$.

\section{RESULTS AND DISCUSSION}

The temperature dependence of the ac conductivity of $\mathrm{Li}_{0.18} \mathrm{La}_{0.61} \mathrm{TiO}_{3}$ is shown in Fig. 1 for several fixed frequencies. It can be observed that at low temperatures experimental conductivity data sets at a given temperature are spaced according to a linear frequency dependence. This linear frequency dependence provides a criterion to determine, at each frequency, a crossover temperature where the nearly constant loss term becomes the dominant contribution to the ac conductivity. At higher temperatures, an additional contribution to the conductivity is observed due to ionic hopping, and the conductivity data become frequency dependent with a sublinear frequency dependence according to Eq. (1). The cross-
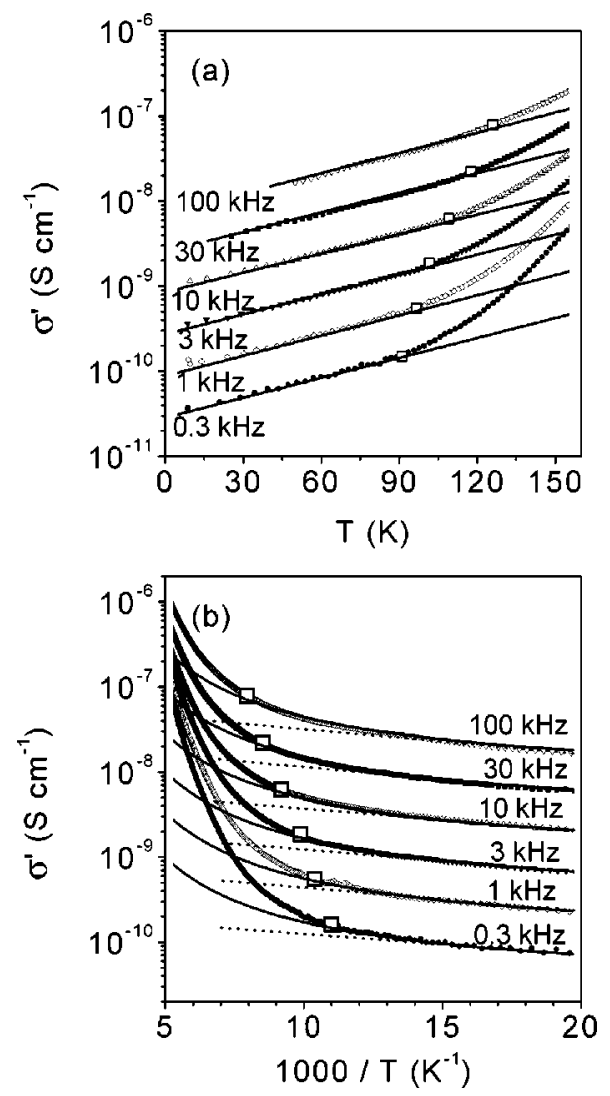

FIG. 1. Conductivity vs temperature plots for $\mathrm{Li}_{0.18} \mathrm{La}_{0.61} \mathrm{TiO}_{3}$. Conductivity data are shown for different frequencies in a linear temperature scale (a) and in an Arrhenius plot (b). Open square symbols represent the crossover between the linear frequencydependent conductivity (nearly constant loss) and the power law regime of Eq. (1) which is due to ionic hopping. Solid lines are fits to an exponential temperature dependence of conductivity data in the nearly constant loss regime. Dotted lines in (b) are fits according to a thermally activated temperature dependence of the ac conductivity at low temperature.

over points, represented by open squares in Fig. 1, were obtained from the temperature where an arbitrarily chosen $10 \%$ departure from linearity was observed. ${ }^{27}$ It is also found that the NCL regime with $\sigma^{\prime}(\omega) \approx A \omega$ is characterized by a weak temperature dependence which can be well described over a wide range at low temperatures by the exponential form $A$ $\propto \exp \left(T / T_{0}\right)$. Solid lines in Fig. 1(a) are fits to an exponential temperature dependence of the ac conductivity at each frequency, giving a value of $60 \pm 3 \mathrm{~K}$ for the parameter $T_{0}$. However, the true temperature dependence of the NCL is not known a priori, and its weak temperature dependence could arise from a thermally activated ion hopping through lowenergy barriers. Since the temperature dependence should be characterized by an activation energy in such a case, in Fig. 1(b) we also plot the ac conductivity data in an Arrhenius representation. Dotted lines in this figure represent fits to an Arrhenius temperature dependence of the form $A$ $\propto \exp (-B / T)$, showing nearly horizontal lines with $B=55$ $\pm 10 \mathrm{~K}$ or activation energies of $5 \pm 1 \mathrm{meV}$. In Fig. 1(b), with solid lines, we also plot the best fits to an exponential temperature dependence of the form $A \propto \exp \left(T / T_{0}\right)$. It can 

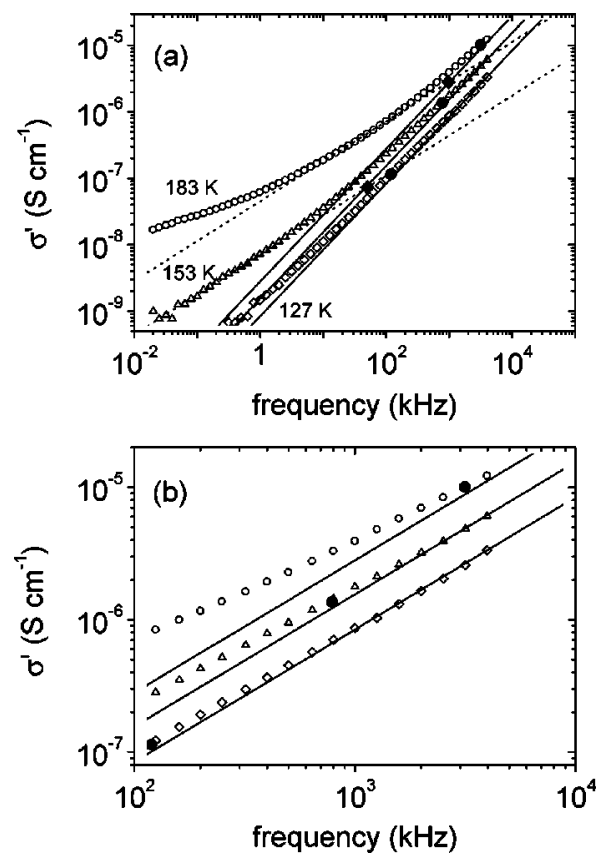

FIG. 2. (a) Frequency dependence of conductivity data showing the crossover between power law term (dotted lines) and NCL (continuous lines) at several temperatures. Solid circles are the crossover points obtained for a $10 \%$ departure from the linear behavior observed at the highest frequencies. Solid diamonds represent the crossover points obtained by assuming the validity of Eq. (2) (see the text). Solid and dashed lines represent the asymptotic linear and power-law frequency dependences of the ac conductivity, respectively. (b) Magnified view illustrating the $10 \%$ deviation from linearity at the crossover.

readily be observed that the latter are hyperbolic curves in the Arrhenius representation and fit the temperature dependence of the ac conductivity over a much wider temperature range than do the Arrhenius fits alone. In fact, the exponential fits account perfectly for the temperature dependence of the ac conductivity at low temperatures up to the crossover points (open squares), which were calculated independently by the $10 \%$ criterion in the frequency dependence. ${ }^{27}$ Note that the crossover points were defined by using a criterion based on the frequency dependence of ac conductivity at a fixed temperature, and therefore without any consideration of the exact temperature dependence of the NCL, which is unknown a priori. It is only a posteriori that we realize that the crossover points seem also to separate two different temperature dependences of the ac conductivity at a fixed frequency. We find an excellent agreement between the temperatures at which our crossover points are obtained and the temperatures at which ac conductivity starts to deviate from an exponential temperature dependence. This result gives further support to our contention that a true crossover exists at these points between a NCL and a power law regime.

Next, in Fig. 2(a) we show the frequency dependence of the ac conductivity of $\mathrm{Li}_{0.18} \mathrm{La}_{0.61} \mathrm{TiO}_{3}$ at several temperatures where a crossover from a power-law dependence to a linear frequency dependence is observed within our experimental frequency window. Solid circles denote the crossover where ac conductivity deviates by $10 \%$ from its linear fre-

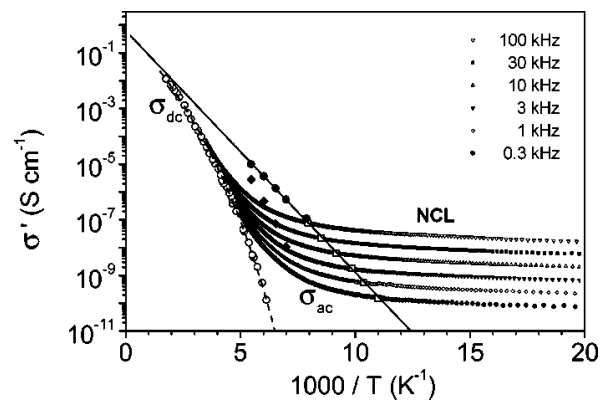

FIG. 3. Arrhenius plots of conductivity data at different frequencies. Open square symbols represent the crossover points obtained from Fig. 1, and solid circles are the crossover points at higher frequencies obtained from Fig. 2. The solid line is a fit to an Arrhenius law for the temperature dependence of the crossover. Open circles are dc conductivity data. Solid diamonds, as in Fig. 2, are the crossover points calculated by assuming the validity of Eq. (2) and show an activation energy close to that observed for the dc conductivity [see Eq. (7) in the text].

quency dependence shown at higher frequencies $\left[\sigma^{\prime}(\omega)\right.$ $=1.1 A \omega]$. A detailed and magnified view of such a crossover is shown in Fig. 2(b). From the procedure shown in Fig. 1 , we have obtained crossover temperatures at frequencies from $100 \mathrm{~Hz}$ to $100 \mathrm{kHz}$. The data shown in Fig. 2 allow us to extend the determination of the crossover temperatures to higher frequencies, from $100 \mathrm{kHz}$ up to $3 \mathrm{MHz}$. Conductivity data at the crossover temperatures, and for each frequency, are plotted in Fig. 3 in an Arrhenius manner. Good agreement is found between the temperature dependence of the crossover points determined from isochronal conductivity measurements in Fig. 1 (open squares), and the temperature dependence of the crossover points from isothermal conductivity curves in Fig. 2 (solid circles). Crossover points show a thermally activated behavior with an activation energy $E$ $=0.17 \pm 0.03 \mathrm{eV}$. Interestingly, this activation energy has the same value as that obtained from NMR spin-lattice relaxation and electrical conductivity relaxation ${ }^{30}$ for the barrier for short-range ion motion $E_{m}=0.17 \pm 0.01 \mathrm{eV}$, i.e., the height of the single ion potential well.

The experimental conductivity data of Fig. 1 are also displayed in Fig. 3, but over a wider temperature range. Note that at sufficiently high temperatures, in the long-range ionic motion regime, all conductivity data at the different fixed frequencies tend to collapse onto a single curve, when the ac conductivity becomes frequency independent $\left(\omega \ll \omega_{o}\right)$ and equal to the dc conductivity value at each temperature [see Eq. (1)]. We also plot the temperature dependence of the dc conductivity in Fig. 3 (open circles). It shows a nonArrhenius behavior, with an activation energy of $E_{m}$ $=0.17 \mathrm{eV}$ at high temperatures, while a larger value, $E_{\sigma}$ $=0.4 \mathrm{eV}$, is found at lower temperatures. This nonArrhenius behavior was also recently found in other fast ionic conductors ${ }^{32-34}$ and has been interpreted in the framework of the coupling model as arising from the additional energy required to overcome ion-ion correlations at low temperatures for the cooperative motion of ions. ${ }^{35}$

In the limit of high temperatures, however, there are no correlations in the ionic motions. Rather, ions hop through 
adjacent potential wells independently of one another, and therefore the dc conductivity is thermally activated with the single ion energy barrier $E_{m}$. Moreover, in this hightemperature regime of uncorrelated ionic motion, the dc conductivity can be described by the Nernst-Einstein equation $\sigma_{0}=\left(N q^{2} d^{2}\right) /\left(6 k_{B} T \tau_{m}\right)$. In this expression, $N$ is the mobile ion concentration, $q$ is the ionic charge, $d$ is the ionic jump distance, $T$ is the temperature, and $\tau_{m}^{-1}=\nu_{0} \exp$ $\left(-E_{m} / k_{B} T\right)$ is the effective microscopic hopping rate determined by the attempt frequency $\nu_{0}$ and the single-ion energy barrier $E_{m}$. Interestingly, we found that the extrapolation to higher temperatures of the solid line in Fig. 3 obtained from the temperature dependence of the crossover between the NCL and the ac conductivity at higher temperatures is well accounted for by this expression. Accordingly, the NCL is observed at a given temperature for frequencies

$$
\omega>\tau_{m}^{-1}=\nu_{0} \exp \left(-E_{m} / k_{B} T\right),
$$

and for a given frequency $\omega$ at temperatures below $E_{m} /\left[k_{B} \ln \left(\nu_{0} / \omega\right)\right]$, where ions have not abandoned their own sites. The solid line in Fig. 3 divides the plot into two temperature regions. For all frequencies $\omega$, the right side is where the temperature falls below the crossover temperature and ions remain within their sites. The left side is the regime when enough thermal energy is available for mobile ions to start to jump over their sites (and subsequently adjacent sites), giving an additional contribution to the ac conductivity, which overshadows the linear frequency term. Thus the results show a clear separation between two processes, the NCL and the ac conductivity from ionic hopping [Eq. (1)]. It is found that the condition $\omega>1000 \omega_{0}$ is fulfilled in the NCL region, which definitely points to a prehopping regime where the ions are not rapidly hopping out of their potentialenergy wells.

At times shorter than $\tau_{m}$, the residence time of an ion, the caged ion can only undergo vibrations within a potentialenergy well that is usually anharmonic. ${ }^{36}$ The thermally activated hopping of an ion out of the potential-energy well is a stochastic process that has a nonzero probability even at times shorter than $\tau_{m}$. Since other ions play a role in defining the potential-energy well for the ion, the small and slowly varying probability of their escape from their own individual wells means that the potential-energy cage is also decaying with time. The cage decay is slow in the time regime of $t \ll \tau_{m}$, and therefore gives rise to a nearly frequency independent loss for the frequency range $\omega \gg 1 / \tau_{m}$.

Such a qualitative description of the NCL has support from molecular-dynamics simulations ${ }^{37,38}$ from the time evolution of the van Hove function at times before the ions leave their cages, where the mean-square displacement was found to increase very slowly with $\log (t)$, consistent with NCL. Such a time dependent mean-square displacement will give rise to the NCL through the relation between the frequency dependence of conductivity and the time dependence of mean-square displacement; ${ }^{39}$

$$
\sigma(\omega) \propto \omega^{2} T^{-1} \lim _{\varepsilon \rightarrow 0} \int_{0}^{\infty} d t<r^{2}(t)>e^{i \omega t-\varepsilon t} .
$$

However, more quantitative discussion of models that actually lead to a NCL (Ref. 40) is beyond the scope of this paper, which is restricted to present experimental evidence of the existence of the crossover.

As a further property of the crossover, we point out that the finding of an activation energy $E=E_{m}=0.17 \mathrm{eV}$ for the conductivity at the crossover from the NCL to a sublinear frequency dependent ac conductivity is at odds with the usual assumption ${ }^{2,21-26}$ that the total ac conductivity in ionic conductors can be described by the augmented Jonscher expression [Eq. (2)]. According to Eq. (2), where the ac hopping ionic conductivity and the NCL contribution are considered to be additive terms, the activation energy of the crossover should be close to the dc conductivity activation energy $E_{\sigma}$ and much higher than $E_{m}$, as observed by experiment. Assuming the validity of Eq. (2), and following the $10 \%$ criterion used in the present work to define the crossover points in Figs. 1 and 2, we should expect that the crossover frequency $\omega_{x}$ at a given temperature satisfies the expression

$$
\sigma^{\prime}\left(\omega_{x}\right)=\sigma_{0}\left[1+\left(\omega_{x} / \omega_{o}\right)^{n}\right]+A \omega_{x}=1.1 A \omega_{x} .
$$

As shown by the experimental data in Fig. 3, at the frequency $\omega_{x}$ the dc conductivity $\sigma_{0}$ makes a negligible contribution to $\sigma^{\prime}\left(\omega_{x}\right)$, so that the conductivity can be written as

$$
\sigma^{\prime}\left(\omega_{x}\right) \approx \sigma_{0}\left(\omega_{x} / \omega_{o}\right)^{n}+A \omega_{x}=1.1 A \omega_{x} .
$$

Solving Eq. (6) for $\omega_{x}$, we obtain

$$
\omega_{x}=\sigma_{0}(0.1 A)^{-1 /(1-n)}\left(\sigma_{0} / \omega_{o}\right)^{n /(1-n)},
$$

and inserting Eq. (7) back into Eq. (6), for the conductivity at the crossover frequency one obtains

$$
\begin{aligned}
\sigma^{\prime}\left(\omega_{x}\right) & =1.1 A \omega_{x} \\
& =1.1 \sigma_{0}(0.1)^{-1 /(1-n)} A^{-n /(1-n)}\left(\sigma_{0} / \omega_{o}\right)^{n /(1-n)} .
\end{aligned}
$$

Since $\sigma_{0}$ and $\omega_{o}$ are thermally activated with the dc activation energy $E_{\sigma}$, it can be shown from Eq. (8) that, assuming a weak temperature dependence of the NCL of the exponential form $\exp \left(T / T_{0}\right)$ (see Fig. 1), the activation energy for the crossover should be

$$
E=-k_{b}\left[d \ln \sigma^{\prime}\left(\omega_{x}\right) / d T^{-1}\right]=E_{\sigma}-[n /(1-n)] k_{b} T^{2} / T_{0},
$$

which is essentially equal to $E_{\sigma}$, provided that $T / T_{0}<1$ and $k_{b} T$ is much smaller than $E_{\sigma}$, which is the case.

The "artificial" crossover of Eq. (5) is obtained graphically in Fig. 2. Note that although we have chosen a $10 \%$ criterion $\left[\sigma^{\prime}\left(\omega_{x}\right)=1.1 A \omega_{x}\right]$ to define the crossover frequency and derive Eq. (9), the result is independent of the criterion of the percentage of deviation from linearity. This is true in particular for $\sigma^{\prime}\left(\omega_{x}\right)=2 A \omega_{x}$, which should hold at the intersection of the assymptotic power law and the linear behaviors of the ac conductivity at low and high frequencies, respectively, assuming the validity of Eq. (2). We have plotted these intersection points in Fig. 2 (solid diamonds), and 
their temperature dependence in Fig. 3 (solid diamonds). As expected from Eq. (9), they show an activation energy close to $E_{\sigma}$, the activation energy obtained for the dc conductivity.

Equation (9), derived from Eq. (2), is unphysical because none of the mechanisms on both sides of the crossover has anything to do with the long-range displacement of ions or its activation energy $E_{\sigma}$. In fact, the experimental data (see Fig. 3) show that the conductivity at this crossover $\left[\sigma^{\prime}\left(\omega_{x}\right)\right]$ is thermally activated, but with a much lower activation energy $E_{m}$ than $E_{\sigma}$, and its value is close to the microscopic energy barrier for a single ion to hop between adjacent wells. Experimental findings are in accord with Eq. (2) as an empirical relation at a given temperature, but the resulting temperature dependence of the parameters are at variance with the observed temperature dependence of the NCL over a wide temperature range. The crossover from the NCL contribution (at times shorter than the microscopic residence time, $\tau_{m}$, of ions in their wells) to the onset of ionic hopping between adjacent wells (at times larger than $\tau_{m}$ ) is more complicated than a simple sum in the frequency domain. Ultimately Eq. (2) implies that the NCL contribution is present even at the lowest frequencies and sufficiently low temperatures, where a steady-state flow with constant dc conductivity has been reached (i.e., the two mechanisms are in parallel). Consequently, the observed conductivity cannot be described simply by a sum, as suggested by Eq. (2), because the sum implies continued existence of the NCL at times (frequencies) much longer (lower) than the residence time (frequency) of the ions in the wells, which is not observed experimentally. The experimental findings suggest a serial picture in which NCL is automatically terminated when the ions have a significant probability to leave their cages and start to hop between adjacent wells; consequently, there is a genuine crossover from NCL to ion hopping ac conductivity.

\section{CONCLUSIONS}

In summary, we have investigated the nature and properties of the crossover between the NCL and the ionic hopping contribution to the ac conductivity in the fast ionic conductor $\mathrm{Li}_{0.18} \mathrm{La}_{0.61} \mathrm{TiO}_{3}$. At low temperatures and high frequencies the ac conductivity shows a NCL response, characterized by a weak exponential temperature dependence, while at high temperatures and low frequencies the ac conductivity is due to ionic hopping. We have found that the conductivity at the crossover $\left[\sigma^{\prime}\left(\omega_{x}\right)\right]$ is thermally activated, and its activation energy is significantly lower than the dc conductivity activation energy. Moreover, the activation energy found for the crossover can be identified with the potential-energy barrier that ions must overcome to leave their sites and start to hop.

We have shown that the ac conductivity cannot be described by the "augmented Jonscher expression" in the whole temperature and frequency range. The "augmented Jonscher expression" assumes that the NCL persists indefinitely down to low frequencies and is an additive contribution to the ion hopping conductivity. It is unsurprising that it cannot be valid because our data show that the NCL crosses over to ionic hopping rather than being a simple sum in the frequency domain.

Within this picture, the mean-square displacement of ions while still caged would not be constant but would increase very slowly with time, providing a possible explanation of the NCL, which is accounted for by an approximate logarithmic time dependence of the mean-square displacement. Since other ions play a role in defining the potential-energy well for the ion, the small and slowly varying probability of their escape from their own individual wells means the potential-energy cage is also decaying with time, which allows the mild time dependence of the mean-square displacement in the NCL regime. However, it is not the intent of this work to develop a theory of the NCL based on these ideas. Instead, the experimental facts presented here are the main emphasis of this work that may guide others to construct theoretical models for the NCL consistent with our experimental findings.

\section{ACKNOWLEDGMENTS}

We thank Dr. A. Várez for sample preparation. C. L. thanks Professor J. Ross Macdonald for extensive and valuable discussions. Financial support from CICYT Grant No. MAT98-1053-C04 is acknowledged. K.L.N. was supported by the ONR.
${ }^{1}$ J. Wong and C. A. Angell, Glass Structure by Spectroscopy (Dekker, New York, 1976).

${ }^{2}$ W. K. Lee, J. F. Liu, and A. S. Nowick, Phys. Rev. Lett. 67, 1559 (1991)

${ }^{3}$ K. L. Ngai, J. Chem. Phys. 110, 10576 (1999).

${ }^{4}$ C. A. Angell, Chem. Rev. 90, 523 (1990).

${ }^{5}$ See the collection of papers in J. Non-Cryst. Solids 131-133 (1991); 172-174 (1994); 235-238 (1998).

${ }^{6}$ K. L. Ngai, J. Non-Cryst. Solids 203, 232 (1996).

${ }^{7}$ B. Roling, A. Happe, K. Funke, and M. D. Ingram, Phys. Rev. Lett. 78, 2160 (1997).

${ }^{8}$ P. Lunkenheimer, A. Pimenov, and A. Loidl, Phys. Rev. Lett. 78, 2995 (1997).
${ }^{9}$ K. L. Ngai and C. T. Moynihan, MRS Bull. 23, 51 (1998).

${ }^{10}$ D. L. Sidebottom, Phys. Rev. Lett. 82, 3653 (1999).

${ }^{11}$ K. L. Ngai and C. León, Phys. Rev. B 60, 9396 (1999).

${ }^{12}$ A. Ghosh and A. Pan, Phys. Rev. Lett. 84, 2188 (2000).

${ }^{13}$ J. C. Dyre and T. B. Schroeder, Rev. Mod. Phys. 72, 873 (2000).

${ }^{14}$ J. R. Macdonald, Phys. Rev. B 63, 052205 (2001).

${ }^{15}$ A. K. Jonscher, Dielectric Relaxation in Solids (Chelsea Dielectric Press, London, 1983)

${ }^{16}$ F. S. Howell, R. A. Bose, P. B. Macedo, and C. T. Moynihan, J. Phys. Chem. 78, 639 (1974).

${ }^{17}$ K. L. Ngai, Comments Solid State Phys. 9, 127 (1979).

${ }^{18}$ K. L. Ngai, Phys. Rev. B 48, 13481 (1993).

${ }^{19}$ K. Funke, Prog. Solid State Chem. 22, 111 (1993). 
${ }^{20}$ J. C. Dyre, J. Appl. Phys. 64, 2456 (1988).

${ }^{21}$ C. Cramer, K. Funke, and T. Saatkamp, Philos. Mag. B 71, 701 (1995).

${ }^{22}$ K. L. Ngai, H. Jain, and O. Kanert, J. Non-Cryst. Solids 222, 383 (1997).

${ }^{23}$ C. León, M. L. Lucia, and J. Santamaria, Phys. Rev. B 55, 882 (1997).

${ }^{24}$ A. S. Nowick, A. V. Vaysleb, and W. Liu, Solid State Ionics 105, 121 (1998).

${ }^{25}$ H. Jain and X. Lu, J. Non-Cryst. Solids 196, 285 (1996).

${ }^{26}$ J. R. Macdonald, J. Chem. Phys. 115, 6192 (2001).

${ }^{27}$ C. León, A. Rivera, A. Várez, J. Sanz, J. Santamaria, and K. L. Ngai, Phys. Rev. Lett. 86, 1279 (2001).

${ }^{28}$ Y. Inaguma, L. Chen, M. Itoh, T. Nakamura, T. Uchida, M. Ikuta, and M. Wakihara, Solid State Commun. 86, 689 (1993).

${ }^{29}$ C. León, M. L. Lucia, J. Santamaria, M. A. Paris, J. Sanz, and A. Várez, Phys. Rev. B 54, 184 (1996).

${ }^{30}$ C. León, J. Santamaria, M. A. Paris, J. Sanz, J. Ibarra, and L. M.
Torres, Phys. Rev. B 56, 5302 (1997).

${ }^{31}$ J. A. Alonso, J. Sanz, J. Santamaria, C. León, A. Várez, and M. T. Fernández, Angew. Chem. Int. Ed. Engl. 39, 619 (2000).

${ }^{32}$ J. Kincs and S. W. Martin, Phys. Rev. Lett. 76, 70 (1996).

${ }^{33}$ K. L. Ngai and A. K. Rizos, Phys. Rev. Lett. 76, 1296 (1996).

${ }^{34}$ P. Maass, M. Meyer, A. Bunde, and W. Dieterich, Phys. Rev. Lett. 77, 1528 (1996).

${ }^{35}$ K. L. Ngai, G. N. Greaves, and C. T. Moynihan, Phys. Rev. Lett. 80, 1018 (1998).

${ }^{36}$ B. Vessal, A. Amini, B. Fincham, and C. R. A. Catlow, Philos. Mag. B 60, 753 (1989).

${ }^{37}$ W. Smith, W. Gillen, and G. N. Greaves, J. Chem. Phys. 103, 3091 (1995).

${ }^{38}$ J. Habasaki, I. Okada, and Y. Hiwatari, Phys. Rev. B 55, 6309 (1997).

${ }^{39}$ M. Lax, Rev. Mod. Phys. 32, 25 (1960).

${ }^{40}$ K. L. Ngai, R. W. Rendell, and C. León, J. Non-Cryst. Solids (to be published). 